\title{
Composición lipídica y caracterización de los ácidos grasos de la semilla de chía (salvia hispánica 1.)
}

\section{Resumen}

Las alteraciones del metabolismo lipídico y la incertidumbre a seleccionar alimentos benéficos para el organismo humano, conducen a la investigación de los ácidos grasos omega. E estudio evalúa el efecto de tres métodos de acondicionamiento de la semilla de chía (Salvia hispánica L.), previo a la extracción del aceite mediante dos métodos y, la caracterización lipídica de semillas provenientes de Colta-Ecuador. El rendimiento de aceite de las semillas acondicionadas presentó diferencias estadísticas. En cuanto al método de extracción, las diferencias fueron altamente significativas, la utilización de eter en Soxhlet mostró mayo rendimiento en una relación aproximada de 2:1 frente a la inmersión en hexano. La presencia y contenido de ácidos grasos se determinó mediante el uso de un cromatógrafo de gases con detector FID marca Agilent Technologies 5975C, los ácidos grasos se separaron con una columna capilar fusionada de sílica SUPELCO 2560 con espesor de película de $100 \mathrm{~m} \times 0,25 \mathrm{~mm}$ i.d. $\times 0,20 \mu \mathrm{m}$. como fase estacionaria; la temperatura del horno fue programada a $140{ }^{\circ} \mathrm{C}$ (5 minutos), hasta $240^{\circ} \mathrm{C}$ ( 15 minutos), se utilizó el inyector a 260 ${ }^{\circ} \mathrm{C}$ con un volumen de inyección de $2 \mu \mathrm{l}$, Split:40:1 y; como fase móvil, el gas portador Helio $20 \mathrm{~cm} / \mathrm{s}$ a $175^{\circ} \mathrm{C}$. Los ácidos grasos mayoritariamente presentes en el aceite fueron linolénico y linoleico, ácidos grasos poliinsaturados de la familia omega. Los resultados obtenidos dejan ver que el aceite de chía constituye un saludable ingrediente 0 aditivo para la elaboración de alimentos funcionales ricos en ácidos grasos omega-3.

Palabras clave: grasa; cromatografía; ácidos grasos; omega-3; chía.

FAT COMPOSITION AND FATTY ACID CHARACTERIZATION OF CHIA SEED (SALVIA HISPÁNICA L.)

\section{Summary}

The alterations of the lipid metabolism and the uncertainty at the moment of selecting beneficial foods to the human body lead to the investigation of omega fatty acids. The study is based on the evaluation of the effect of three preparation methods of chía seed, before getting the oil through two extraction methods and the characterization of the lipid fraction of chia seed (Salvia hispánica L.) from Colta-Ecuador. The oil yield of the conditioned seeds showed statistical differences. Regarding the extraction method, the differences were highly significant, the use of ether in the Soxhlet equipment showed the superiority in the oil performance in a relation of approximately 2:1 versus immersion in hexane. The presence and the content of fatty acids was determined by the use of a gas chromatograph with FiD detector Agilent Technologies $5975 \mathrm{C}$ brand, fatty acids were separated with a capillary column fused of silica SUPELCO 2560 with film thickness of $100 \mathrm{~m} \times 0,25$ $\mathrm{mm}$ i.d. $\times 0,20 \mu \mathrm{m}$ as a stationary phase; the oven temperature was programmed from $140{ }^{\circ} \mathrm{C}$ (5 minutes), to $240{ }^{\circ} \mathrm{C}$ ( 15 minutes), the injector was used at $260^{\circ} \mathrm{C}$ with injection volume of $2 \mu$, Split:40:1 and the Helium carrier gas $20 \mathrm{~cm} / \mathrm{s}$ at $175^{\circ} \mathrm{C}$ as a mobile phase. Fatty acids predominantly presented in the oil were linolenic and linoleic, polyunsaturated fatty acids of the omega family. The results obtained revealed that chia oil is a healthy ingredient or additive for the elaboration of the functional foods rich in omega- 3 fatty acids.

Keywords: fat; chromatography; fatty acids; omega-3; chía.

\section{INTRODUCCIÓN}

La preocupación por los aspectos de salud y seguridad de los aceites y productos grasos que se utilizan en la dieta, han conducido al desarrollo de técnicas y procedimientos analíticos que permitan obtener información detallada acerca de la naturaleza, el carácter, el tratamiento y la composición de los citados nutrientes (Kirk, Sawyer y Egan, 2011). El presente estudio tuvo el objetivo de evaluar el efecto de dos métodos de extracción de aceite a partir de semillas acondicionadas por secado, liofilización y en fresco y la posterior caracterización y cuantificación de los ácidos grasos presentes en la semilla de chía. Debido a importantes beneficios y al gran aporte para la industria alimentaria (González, Kafarov, y Guzmán, 2009), la semilla de chía es un alimento recomendado para el consumo por la presencia de factores nutricionales como los ácidos grasos omega- 3 y aminoácidos esenciales que favorecen el adecuado funcionamiento del organismo humano (Salazar, 2009).

La cantidad de lípido extraído de una materia alimentaria dependerá del contenido presente por naturaleza y del método utilizado para su extracción. La obtención del extracto etéreo se ha realizado a través de técnicas disponibles para determinar de forma rápida el porcentaje de grasa, las mismas incluyen aquellas técnicas empleadas para el procesamiento de alimentos (Kirk, et al. 2011). La extracción de la fracción lípidica es posible gracias a la alta solubilidad de las grasas en solventes orgánicos no polares como éter, benceno, hexano y otros afines (Luna, 2007). Hoy en día, el equipo de cromatografía de gases y el uso de columnas capilares, con gran poder de resolución, permiten conocer la composición posicional de los triglicéridos y composición isomérica geométrica de los ácidos grasos (Kirk, et al. 2011).

La importancia de los mencionados nutrientes radica en sus múltiples efectos biológicos, cuyas evidencias reportan variados mecanismos cardioprotectores relacionados con la mejora del perfil lipídico (Villalobos, Sánchez-Muniz, Acín, Vaquero, Higueras y Bastida, 2010). Sin embargo, el balance de los ácidos grasos de las series omega-9 (oleico), omega-6 (linoleico) y omega-3 (lino-

Docente Ingeniería Agroindustrial y de Alimentos. Universidad de las Américas. Ecuador. maria. mosquera@udla.edu.ec

2 Estudiante Ingeniería Agroindustrial y de Alimentos. Universidad de las Américas. Ecuador. mjreinoso@udlanet.ec

3 Docente Ingeniería Agroindustrial y de Alimentos. Universidad de las Américas. Ecuador. wilson.vasquez@udla.edu.ec 
lénico) constituye un factor de vasta importancia y necesaria investigación al momento de aprovechar el efecto sinérgico entre ácidos grasos e inclusive la sinergia con otros componentes que limitan o potencian su acción.

\section{MATERIALES Y MÉTODOS}

La semilla de chía utilizada en el estudio fue producida en el cantón Colta ubicada en la provincia de Chimborazo, Ecuador, a 2758 msnm con una temperatura promedio de $14^{\circ} \mathrm{C}$ y precipitación anual de $561 \mathrm{~mm}$. La cantidad inicial de semilla utilizada, previo a la extracción del aceite, fue de 100g; esta fue acondicionada por tres métodos: 1) desecación, 2) liofilización y 3) en fresco. La extracción del aceite de la semilla se realizó utilizando dos metodologías, extracción por inmersión en 1) hexano y extracción con 2) éter en Soxhlet.

La caracterización y cuantificación de ácidos grasos se realizó en el laboratorio de Nutrición y Calidad de la Estación Santa Catalina del Instituto Nacional de Investigaciones Agropecuarias (INIAP). EI equipo utilizado fue un cromatógrafo de gases con detector FID marca Agilent Technologies 5975C, los ácidos grasos fueron separados con una columna capilar fusionada de sílica SUPELCO 2560 con espesor de película de $100 \mathrm{~m}$ x 0,25 mm i.d. x 0,20 $\mu \mathrm{m}$. como fase estacionaria; la temperatura del horno fue programada a $140{ }^{\circ} \mathrm{C}$ (5 minutos), hasta 240 ${ }^{\circ} \mathrm{C}$ (15 minutos), se utilizó el inyector a $260{ }^{\circ} \mathrm{C}$ con un volumen de inyección de $2 \mu \mathrm{l}$, Split:40:1 y; como fase móvil, el gas portador Helio $20 \mathrm{~cm} / \mathrm{s}$ a $175^{\circ} \mathrm{C}$. Las muestras fueron obtenidas del aceite de las semillas acondicionadas por liofilización y extraído mediante el uso del solvente éter; cada muestra fue tratada para convertir los ácidos grasos en ésteres metílicos derivatizados para su posterior análisis por cromatografía de gases.

El tipo de investigación fue experimental y el análisis estadístico se realizó utilizando un diseño de bloques completos al azar en arreglo factorial $3 \times 2$ para el rendimiento de aceite; las variables analizadas fueron: tipo de acondicionamiento de la semilla (secado, liofilizado y en fresco) y tipo de extracción de aceite (por inmersión en hexano y con éter en Soxhlet). Para la caracterización del aceite, se utilizó un diseño de bloques completos al azar; estos análisis fueron evaluados en el programa estadístico InfoStat/L.

\section{RESULTADOS Y DISCUSIÓN}

El análisis de varianza permitió determinar diferencias estadísticas entre los métodos de extracción de aceite de la semilla de chía. El método de ex- tracción con éter en Soxlet fue más efectivo por presentar $15.66 \%$ más volumen de aceite frente al método de inmersión en hexano como se muestra en la Tabla 1.

Tabla 1. Promedio de la cantidad de grasa (\%) de la semilla de chía a través de dos métodos de extracción, Colta, 2015

\begin{tabular}{|c|c|}
\hline Solvente & Grasa (\%) \\
\hline Éter & $32.94 \pm 0.7 \mathrm{a}$ \\
\hline Hexano & $17.28 \pm 1.0 \mathrm{~b}$ \\
\hline
\end{tabular}

Fuente: Elaboración propia

El análisis estadístico permitió determinar la gran efectividad del solvente éter respecto del volumen de aceite obtenido en el proceso de extracción frente al tratamiento de inmersión en hexano.

El solvente éter fue más efectivo para la extracción total de grasa por alcanzar mayor eficiencia dado su bajo punto de ebullición $35^{\circ} \mathrm{C}$ y mayor volatilidad, esto permitió un rendimiento óptimo de grasa, $99 \%$ y un volumen de aceite correspondiente al $32.94 \%$. Los resultados obtenidos concuerdan con estudios realizados por Yxtaina (2010), quien obtuvo un $33,6 \%$ de grasa.

Es importante indicar que el tiempo de extracción no debe ser superior a $8 \mathrm{~h}$ ya que, pasado este tiempo, se extraen componentes no lipídicos. Por otro lado, si el tiempo es menor, la extracción es incompleta como indica la investigación realizada por Quintana y Valencia (2014) quienes obtuvieron sólo $14 \%$ en rendimiento de aceite en un tiempo de $4 \mathrm{~h}$. En este sentido, el solvente éter, ofrece el mejor balance de propiedades físico químicas como: mayor límite de saturación y mayor selectividad respecto al soluto por extraer, baja presión de vapor, baja viscosidad, baja toxicidad, baja densidad, baja tensión superficial, baja inflamabilidad y bajo costo, siendo más selectivo para lípidos hidrofóbicos (Núñez 2008).

En la interacción entre los tipos de semilla (acondicionamiento previo a la extracción) y tipo de solvente, se encontraron diferencias significativas como se aprecia en la Tabla 2.

Tabla 2. Promedio de la cantidad de grasa (\%) de la semilla de chía de la interacción entre los métodos de extracción de grasa y tipo de semilla, Colta, 2015.

\begin{tabular}{|c|c|c|}
\hline Tipo de semilla & Solvente & Grasa (\%) \\
\hline Seca & Éter & $33.66 \pm 1.0 \mathrm{a}$ \\
\hline Fresca & Éter & $32.83 \pm 0.2 \mathrm{a}$ \\
\hline Liofilizada & Éter & $32.32 \pm 3.2 \mathrm{a}$ \\
\hline Fresca & Hexano & $17.95 \pm 1.4 \mathrm{~b}$ \\
\hline Liofilizada & Hexano & $17.74 \pm 0.4 \mathrm{~b}$ \\
\hline Seca & Hexano & $16.15 \pm 2.9 \mathrm{~b}$ \\
\hline
\end{tabular}

Fuente: Elaboración propia 
El análisis estadístico permitió determinar la correlación existente entre los tipos de semilla y el solvente utilizado para la extracción de aceite. Con el uso del solvente éter, se obtuvo los volúmenes más altos de aceite, las semillas acondicionadas por secado fueron las de mayor rendimiento con $33.66 \%$, seguidas de las semillas procesadas en fresco con $32.83 \%$ y finalmente las semillas liofilizadas con $32.32 \%$ de aceite. En el caso del solvente hexano, los resultaron fueron: $17.95 \%$ para las semillas en fresco, 17.74 $\%$ para las semillas liofilizadas y $16.15 \%$ para las semillas secas. Estos resultados se corroboran con el estudio realizado por Marín, Larrea, Navarro y Arozarena, (2014) quienes estudiaron el comportamiento de semillas de uva sometidas a varios métodos de secado previo a la extracción de componentes, en los resultados obtenidos en cuanto al contenido de polifenoles totales, no aparecieron diferencias significativas entre los extractos hasta que se trabajó a temperaturas superiores a $50{ }^{\circ} \mathrm{C}$.

La efectividad del aislamiento o separación de componentes de las semillas comporta el conocimiento del grado de influencia de los métodos de preparación de las mismas. En el estudio previo, este comportamiento es independiente del pretratamiento de secado utilizado. Sin embargo, se observó que, al trabajar con mayores temperaturas de secado, el rendimiento obtenido en polifenoles totales fue mayor en el caso de las pepitas liofilizadas; en dicho estudio se concluyó que, el tratamiento de secado por liofilización permite una mayor riqueza en polifenoles y mayor poder antioxidante, componentes analizados en el estudio mencionado, Marín, et al (2014).

El secado, y el debido manejo de factores como la temperatura y el tiempo se ha aplicado en gran parte de los casos estudiados, así, se ha realizado dicho tratamiento en hornos convencionales y secado a vacío, sin embargo, poco se ha utilizado, con este propósito, la tecnología de la liofilización, Pinelo, Sineiro y Nuñez, (2004), Makris, (2007). Los resultados obtenidos en el presente estudio dejan ver que, para la separación de componentes adicionales a la grasa como mucílago, proteína, fibra, entre otros, la preparación de la semilla previo a la extracción, presenta gran influencia dados los altos rendimientos de grasa con el uso de solvente éter, a partir de semillas acondicionadas por liofilización.

Respecto de la identificación y cuantificación de ácidos grasos mediante la técnica de cromatografía de gases, se identificó a los ácidos grasos de la familia omega como los más abundantes. En la evaluación de los citados componentes, el análisis de varianza reportó diferencias significa- tivas como se observa en la Tabla 3.

Tabla 3. Promedio de la cantidad de ácidos grasos del aceite de semilla de chía en 100g., Colta, 2015

\begin{tabular}{|l|c|}
\hline \multicolumn{1}{|c|}{ Ácidos Grasos } & Ácidos (\%) \\
\hline Ácido miŕstico & $0.06 \pm 0.01 \quad \mathrm{jk}$ \\
\hline Ácido pentadecanoico & $0.02 \pm 0.01 \mathrm{~lm}$ \\
\hline Ácido palmítico & $7.54 \pm 0.06 \quad \mathrm{c}$ \\
\hline Ácido palmitoleico & $0.01 \pm 0.00 \mathrm{~m}$ \\
\hline Ácido heptadecanoico & $0.05 \pm 0.02 \quad \mathrm{kl}$ \\
\hline Ácido cis-10- heptadecanoico & $0.01 \pm 0.01 \quad \mathrm{~m}$ \\
\hline Ácido estearico & $3.47 \pm 0.02 \quad \mathrm{e}$ \\
\hline Ácido oleico & $6.07 \pm 0.01 \quad \mathrm{~d}$ \\
\hline Ácido linoleico & $21.66 \pm 0.02 \quad \mathrm{~b}$ \\
\hline Ácido araquídico & $0.27 \pm 0.01 \quad \mathrm{~g}$ \\
\hline Ácido r- linoleico & $0.38 \pm 0.01 \quad \mathrm{f}$ \\
\hline Ácido-cis-11-eicosenoico & $0.17 \pm 0.01 \quad \mathrm{~h}$ \\
\hline Ácido linolénico & $57.55 \pm 0.07 \quad \mathrm{a}$ \\
\hline $\begin{array}{l}\text { Ácido cis-11-14 } \\
\text { eicosadienoico }\end{array}$ & $0.04 \pm 0.01 \mathrm{klm}$ \\
\hline Ácido behénico & $0.08 \pm 0.02 \quad \mathrm{j}$ \\
\hline Ácido erucico & $0.02 \pm 0.01 \mathrm{Im}$ \\
\hline $\begin{array}{l}\text { Ácido cis-11,14,17 } \\
\text { eicosatrienoico }\end{array}$ & $0.06 \pm 0.00 \mathrm{jk}$ \\
\hline Ácido tricosanoico & $0.02 \pm 0.01 \quad \mathrm{~mm}$ \\
\hline $\begin{array}{l}\text { Ácido cis 13,16- } \\
\text { docosadienoico }\end{array}$ & $0.01 \pm 0.00 \mathrm{~m}$ \\
\hline Ácido lignoserico & $0.12 \pm 0.01 \quad \mathrm{i}$ \\
\hline Ácido nervorico & $0.01 \pm 0.01 \mathrm{~m}$ \\
\hline
\end{tabular}

Fuente: Elaboración propia

A través de un diseño de bloques completos al azar para el análisis de los resultados obtenidos de la corrida de muestras de aceite de chía previamente tratadas, se observó que la semilla contiene altos porcentajes de ácidos grasos poliinsaturados. Estos datos concuerdan con los obtenidos en el estudio de Quintana y Valencia (2014) quienes reportan la presencia de $61,55 \%$ de ácido linolénico y $14.42 \%$ de ácido linoleico. Los resultados obtenidos en el presente estudio indican que los principales ácidos grasos detectados en el aceite de la semilla de chía en orden de abundancia fueron el ácido linolénico (omega-3) en una proporción de $57.55 \%$, ácido linoleico (omega-6) en $21.66 \%$ y ácido oleico (omega-9) en una proporción de $6.07 \%$. La Tabla 4, muestra los tiempos de retención transcurridos de cada uno de los ácidos grasos, desde el ingreso a la columna hasta la respectiva elución al detector, parámetro que permitió la detección de la calidad y cantidad de los ácidos grasos presentes. 
Tabla 4. Tiempos de retención de los ácidos grasos de las muestras esterificadas del aceite de semilla de chía, Colta, 2015.

\begin{tabular}{|l|c|}
\hline \multicolumn{1}{|c|}{ Ácidos Grasos } & $\begin{array}{c}\text { Tiempos de retención } \\
\text { (segundos) }\end{array}$ \\
\hline Ácido mirístico & 19.825 \\
\hline Ácido pentadecanoico & 21.464 \\
\hline Ácido palmítico & 23.106 \\
\hline Ácido palmitoleico & 24.342 \\
\hline Ácido heptadecanoico & 24.703 \\
\hline Ácido cis-10- heptadecanoico & 25.914 \\
\hline Ácido estearico & 26.206 \\
\hline Ácido oleico & 27.304 \\
\hline Ácido linoleico & 28.802 \\
\hline Ácido araquídico & 29.202 \\
\hline Ácido r- linoleico & 29.909 \\
\hline Ácido-cis-11-eicosenoico & 30.165 \\
\hline Ácido linolénico & 30.41 \\
\hline Ácido cis-11-14 eicosadienoico & 31.613 \\
\hline Ácido behénico & 31.982 \\
\hline Ácido erúcico & 32.959 \\
\hline Ácido cis-11,14,17 eicosatrienoico & 33.304 \\
\hline Ácido tricosanoico & 33.377 \\
\hline Ácido cis 13,16- docosadienoico & 34.459 \\
\hline Ácido lignoserico & 34.828 \\
\hline Ácido nervorico & 35.886 \\
\hline
\end{tabular}

Fuente: Elaboración propia

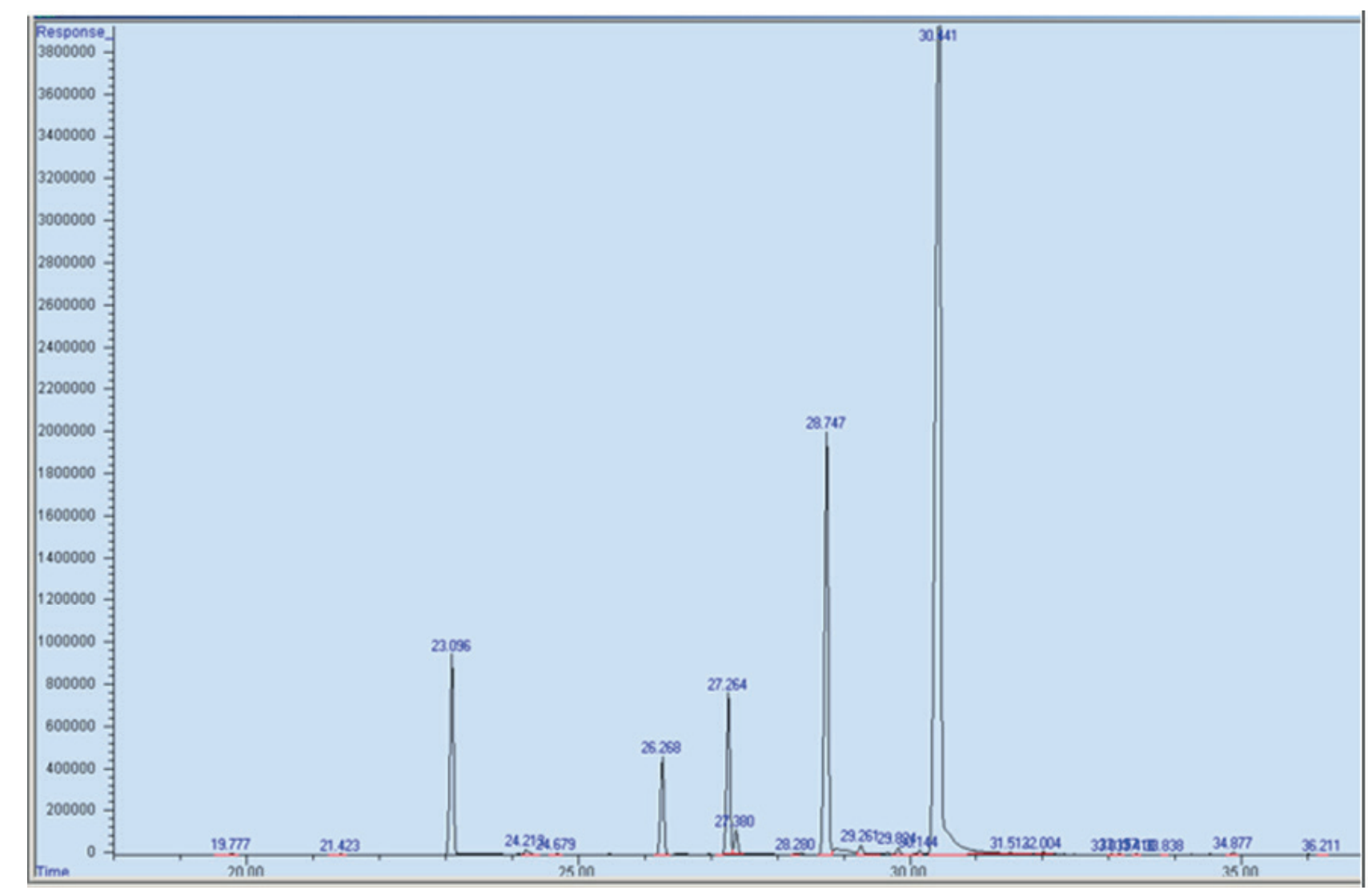

Figura 1. Cromatograma de ácidos grasos del aceite de la semilla de chía, Colta 2015.
Con base en los tiempos de retención, se generó un cromatograma que mostró el tipo y grado de concentración en que están presentes los ácidos grasos según el análisis del área de pico, correspondiente a la cantidad de ácidos grasos presentes en la muestra, evaluadas en función del tiempo transcurrido desde la inyección de la muestra hasta que el pico llegó a la máxima altura. Los datos almacenados en el sistema, dibujaron el cromatograma como muestra la Figura 1.

Resultados obtenidos en estudios relacionados, muestran que el principal ácido graso presente en el aceite de semilla de chía es el ácido linolénico con 61-74 \% (Quintana y Valencia, 2014), estos porcentajes concuerdan con estudios realizados previamente por Ayerza, (2009) cuyos resultados revelaron la presencia de ácido linolénico en 64$69 \%$, cantidad que fue detectada en el análisis del aceite de semilla de chía obtenido mediante la aplicación de metodología mecánica, el prensado.

El volumen de aceite de la semilla de chía y la composición de ácidos grasos puede variar de acuerdo a la influencia producida por factores ambientales de la localidad del cultivo, así, la presencia de ácido linolénico en algunos países (Argentina $63.1 \%$, Bolivia $63.6 \%$, Colombia $57.9 \%$, México, $61.6 \%$, y Perú $64.2 \%$ ) presenta diferencias en función de la 
incidencia de dichos factores como indica Ayerza y Coates (2005); por tanto, el resultado obtenido del volumen de ácido linolénico en la semilla de chía cultivada en Ecuador $57,55 \%$ se encuentra dentro de los parámetros citados anteriormente. Al comparar los resultados obtenidos con los de investigaciones realizadas por Yxtaina, (2010), los porcentajes de ácidos grasos de aceites extraídos por solvente en las localidades de Argentina y Guatemala son menores con excepción del ácido linolénico. Estos resultados concuerdan con estudios realizados previamente por Coates (2012), Ayerza (2009), Association of Oficial Analytical Chemestry (2016), Yxtaina, (2010) y, Quintana y Valencia, (2014).

\section{CONCLUSIONES}

La obtención de aceite de la semilla de chía y su rendimiento en volumen es un factor que está directamente relacionado con el método de extracción aplicado. Se concluye que la extracción con solvente éter, permite obtener un mayor rendimiento de la fracción grasa de la semilla frente al uso del solvente hexano; esta comparación resulta en un indicativo para aprovechar la mayor cantidad de aceite presente en las semillas en función del objetivo científico, tecnológico e industrial.

La interacción de factores como el método de extracción y el acondicionado de la semilla, previo a la obtención de aceite, son variables relacionadas de forma directa. Por tanto, el volumen y la calidad de aceite están en función del tipo de acondicionamiento al cual se somete a la semilla. El análisis de variables adicionales que influyen en el rendimiento de la materia grasa total, como el tamaño de la semilla, la composición bioquímica y las estructuras fisiológicas, son factores a tomar en cuenta al momento de evaluar los componentes nutricionales en los diferentes campos de interés.

La caracterización y cuantificación de los componentes lipídicos presentes en el aceite reveló vasta presencia de ácido linolénico (omega-3) lo que confiere a la semilla un valor biológico superior frente a otros pseudocereales como quínoa y amaranto por ser un ácido esencial y precursor de factores nutricionales adicionales como eicosapentaenoico (EPA) y docosahexaenoico (DHA), ácidos grasos semiesenciales, importantes para el desarrollo neuronal humano.

\section{REFERENCIAS BIBLIOGRÁFICAS:}

[1] Association of Oficial Analytical Chemestry. (2016). Official Method and recommended practices of the American Oil Chemists Society. (20a ed.). Meryland: AOAC International.

[2] Ayerza, R., y Coates, W. (2005). Ground chia seed and chia oil effects on plasma lipids and fatty acids in the rat. Nutrition Research, 25(11), 995-1003. Recuperado de http:// www.sciencedirect.com/science/article/pii/ S0271531705001855

[3] Ayerza, R. (2009). The seed's protein and oil content, fatty acid composition, and growing cycle length of a single genotype of chia ( $\mathrm{Sal}$ via hispanica L.) as affected by environmental factors. J Oleo Sci. 58(7), 347-54. Recuperado de https://www.jstage.jst.go.jp/article/ jos/58/7/58_7_347/_pdf

[4] Coates, W. (2012). Chia: The complete Guide to the Ultimate Superfood. New York: Sterling.

[5] González, A., Kafarov, V., Guzmán, A. (2009). Desarrollo de métodos de extracción de aceite en la cadena de producción de biodiesel a partir de microalgas. Prospect, 7(2), 53-60. Recuperado de http://hdl.handle.net/11619/1348

[6] Kirk, R., Sawyer, R. y Egan, H. (2011). Composición y Análisis de Alimentos. (2a ed.). México: Compañía Editorial Continental, S. A.

[7] Makris, D. (2007). Nuts \& Seeds in Healh and Disease Prevention. San Diego, USA: Elsevier.

[8] Marin, M., Larrea, J., Navarro, M. y Arozarena, I. (2014). Comprobación de métodos de pre- tratamiento de las pepitas de uva para su aprovechamiento en la obtención de extractos polifenólicos con elevada actividad antioxidante. CIBIA9. DTA, 1(13), 185-190. Recuperado de https://riunet.upv. es/bitstream/handle/10251/38245/CIBIA\%209 CONGRESO $\% 20 I B E R O A M E R I C A N O \% 20 D E \% 20$ INGENIER\%CDA\%20DE\%20ALIMENTOS_ LIBRO\%20DE\%20ACTAS_1.pdf?sequence=1

[9] Nuñez. E. (2008). Extracciones con equipo Soxhlet. 15, 2-5. Recuperado de http://www. cenunez.com.ar/archivos/39-ExtraccinconequipoSoxhlet.pdf.

[10] Pinelo, M., Sineiro J. y Nuñez M.J. (2004). Extracción de antioxidantes a partir de residuos de uva: Influencia del modo de operación y de las variables de proceso. (Tesis doctoral). Escuela Técnica Superior de Ingeniería. Universidad Santiago de Compostela. España. 
[11] Quintana, J. y Valencia. J. (2014). Caracterización del aceite de semilla de chía (Salvia hispánica L.) extraído con solvente orgánico y prensado en frío. Ingeniería: Ciencia, Tecnología e Innovación. 2(1). Recuperado de http:// revistas.uss.edu.pe/index.php/ING/article/ view/102

[12] Salazar, I. (2009). Composición en ácidos grasos alfa linolenico $(\omega-3)$ en huevo y carne de aves empleando chía (Salvia hispanica L.) en el alimento. Interciencia, 34(3). DOI 03781844/09/03/209-04.

[13] Villalobos, M., Sánchez-Muniz, F. J., Acín, M., Vaquero, M., Higueras, F. J., y Bastida, S. (2010). Similitudes, diferencias y antagonis- mos en los efectos pleiotrópicos de las estatinas y los ácidos grasos omega-3. Nutrición Hospitalaria, 25(6), 889-909. Recuperado de http://scielo.isciii.es/scielo.php?script=sci_arttext\&pid=S0212-16112010000600003.

[14] Yxtaina, V. (2010). Caracterización de la semilla y aceite de chía (Salvia hispanica L.) obtenido mediante distintos procesos: aplicación y tecnología de alimentos. (Tesis doctoral). Universidad Nacional de la Plata. 